\title{
Study of Adaptive Nulling Methods under Different Constraints
}

\author{
SU Chengxiao ${ }^{1}$, WANG Jiegui ${ }^{1}$, LIU Kai ${ }^{1}$ \\ Electronic Engineering Institute \\ Hefei, P.R.China \\ scx123168@163.com
}

\author{
LIU Songyang ${ }^{2}$ \\ College of Instrumentation \& Electrical Engineering \\ Jilin University \\ Changchun, P.R.China
}

\begin{abstract}
Adaptive nulling methods under multiple constraints for Digital Beamforming (DBF) of Uniform Linear Arrays (ULA) are proposed, which is studied in three aspects: with main lobe constraint only, with main and side lobe constraints, with main and side lobe constraints and given nulls constraints. Optimum weight vector of DBF is derived by Lagrange multiplier approach and calculated with sample matrix inversion algorithm. Effectiveness and correctness of the proposed methods are verified by computer simulations.
\end{abstract}

\section{Key words-DBF; adaptive nulling; constraint}

\section{INTRODUCTION}

Adaptive Digital Beamforming $(\mathrm{ADBF})^{[1]}$ is an advanced array signal processing technique, which is developing from antenna theory, control theory and adaptive signal processing theory. Currently, DBF is mostly used in receiving mode, many radar experts concentrate on the receiving digital beamforming. According to the signal environment, receiving $\mathrm{ADBF}$ is to automatically adjust null to restrain interference ${ }^{[2,3]}$.

Theoretically, transmitting DBF is possible since receiving $\mathrm{DBF}$ is practicable. In recent years, experts have paid more attention to the transmitting DBF and tried to apply it to radar and radar EW system. To radar and radar EW system, adaptive nulling[4,5] of transmitting DBF is very important, which can raise the anti-reconnaissance ability, promote the anti-ARM ability, and avoid the interference of the system.

Adaptive nulling methods with multiple constraints for transmitting DBF of ULA are discussed in the following three circs, with main lobe constraint only, with main and side lobe constraints, with main and side lobe constraints and given nulls constraint. And the optimal weight vector of transmitting DBF is derived by Lagrange multiplier approach and calculated with sample matrix inversion algorithm. The computer simulation result shows that the proposed method is practicable and effective.

\section{ADAPTIVE NULLING METHODS OF DBF}

\section{A. The array signal model}

Suppose that the signal is narrowband, the uniform linear array is composed of $\mathrm{L}$ elements and the space between is $\mathrm{d}$. Take the first array element of the left side as the phase reference. On the condition that there are $\mathrm{m}$ emitters, each emitter is statistically independent, then the signal of element $k$ is

$$
x_{k}(t)=\sum_{m=1}^{M} s_{m}(t) e^{j(k-1) \rho_{r} d \sin \theta_{m}}+n_{k}(t)
$$

where $\rho_{r}=2 \pi / \lambda_{r}, \lambda_{r}$ is the wavelength of the signal, $\theta_{m}$ is the azimuth of emitter $m, n_{k}(t)$ is the measure noise. The matrix form of the equation is

$$
\mathbf{x}(\mathbf{t})=\operatorname{As}(\mathbf{t})+\mathbf{n}(\mathbf{t})
$$

where

$$
\begin{aligned}
& \mathbf{x}(\mathbf{t})=\left[\begin{array}{llll}
x_{1}(t) & x_{2}(t) & \cdots & x_{L}(t)
\end{array}\right]^{T} \text { is the measure vector, } \\
& \mathbf{s}(\mathbf{t})=\left[\begin{array}{llll}
s_{1}(t) & s_{2}(t) & \cdots & s_{M}(t)
\end{array}\right]^{T} \text { is the signal vector, } \\
& \mathbf{n}(\mathbf{t})=\left[\begin{array}{llll}
n_{1}(t) & n_{2}(t) & \cdots & n_{L}(t)
\end{array}\right]^{T} \text { is the noise vector, } \\
& \mathbf{A}=\left[\begin{array}{llll}
a\left(\theta_{1}\right) & a\left(\theta_{2}\right) & \cdots & a\left(\theta_{M}\right)
\end{array}\right]^{T} \text { is the steering matrix, } \\
& a\left(\theta_{m}\right)=\left[\begin{array}{llll}
1 & e^{j \rho_{r} d \sin \theta_{m}} & \cdots & e^{j(L-1) \rho_{r} d \sin \theta_{m}}
\end{array}\right]^{T} \text { is the }
\end{aligned}
$$

steering vector of array element $m$.

The output of the array is the weighted sum of all the element's signal. Assumed that the weight coefficient of the $k$ th element is $w_{k}$, the weight coefficient vector is $\mathbf{w}=\left[\begin{array}{llll}w_{1} & w_{2} & \cdots & w_{L}\end{array}\right]^{T}$, then the output of the array is

$$
y(t)=w^{H} x(t)
$$

And the array pattern is

$$
F(\theta)=\mathbf{w}^{\mathbf{H}} \mathbf{a}(\boldsymbol{\theta})
$$

The power output of the array is $\mathbf{E}\left\{\mathbf{y}(\mathbf{t}) \mathbf{y}^{\mathbf{H}}(\mathbf{t})\right\}=\mathbf{w}^{\mathbf{H}} \mathbf{A} \mathbf{R}_{\mathbf{s}} \mathbf{A}^{\mathbf{H}} \mathbf{w}+\mathbf{w}^{\mathbf{H}} \mathbf{R}_{\mathbf{n}} \mathbf{w}, \quad$ where $\mathbf{R}_{\mathbf{s}}=\mathbf{E}\left\{\mathbf{s}(\mathbf{t}) \mathbf{s}^{\mathbf{H}}(\mathbf{t})\right\}$ is the covariance matrix of the signal, $\mathbf{R}_{\mathbf{n}}=\mathbf{E}\left\{\mathbf{n}(\mathbf{t}) \mathbf{n}^{\mathbf{H}}(\mathbf{t})\right\}$ is the covariance matrix of the noise.

$B$. Adaptive nulling with main lobe constraint

Suppose that all the emitters in equation (2) are interference source (that is $\mathbf{J}(\mathbf{t})=\mathbf{s}(\mathbf{t}), \mathbf{A}_{\mathbf{J}}=\mathbf{A}$ ), and the needing signal is nonexistent, but the direction of the needing signal is known. Then the power out (the power of the interference and noise) is

$$
\mathbf{E}\left\{\mathbf{y}(\mathbf{t}) \mathbf{y}^{\mathbf{H}}(\mathbf{t})\right\}=\mathbf{w}^{\mathbf{H}} \mathbf{A}_{\mathbf{J}} \mathbf{R}_{\mathbf{J}} \mathbf{A}_{\mathbf{J}}^{\mathbf{H}} \mathbf{w}+\mathbf{w}^{\mathbf{H}} \mathbf{R}_{\mathbf{n}} \mathbf{w}
$$

where $\mathbf{R}_{\mathbf{J}}=\mathbf{E}\left\{\mathbf{J}(\mathbf{t}) \mathbf{J}^{\mathbf{H}}(\mathbf{t})\right\}$ is the covariance matrix of interference.

The main lobe constraint is shown as follow.

$$
\mathbf{w}^{\mathbf{H}} \mathbf{a}\left(\theta_{m}\right)=1
$$

where $\theta_{m}$ is the direction of the needing signal, that is, the direction of the main lobe .

Given $\mathbf{R}_{\mathbf{I}}=\mathbf{A}_{\mathbf{J}} \mathbf{R}_{\mathbf{J}} \mathbf{A}_{\mathbf{J}}^{\mathbf{H}}+\mathbf{R}_{\mathbf{n}}$. Since the needing signal is nonexistent, then obviously $\mathbf{R}_{\mathbf{x}}=\mathbf{R}_{\mathbf{I}}$, where 
$\mathbf{R}_{\mathbf{x}}=\mathbf{E}\left\{\mathbf{x}(\mathbf{t}) \mathbf{x}^{\mathbf{H}}(\mathbf{t})\right\}$. As a result, the above-mentioned optimum problem with constraint can be described as: to obtain the optimum weight vector $w$, which satisfies that the power output $\mathbf{P}=\mathbf{w}^{\mathbf{H}} \mathbf{R}_{\mathbf{x}} \mathbf{w}$ reaches minimum value with the constraint (6).

Now we solve the problem with the Lagrange multiplier approach, the Lagrange function is defined as follow.

$$
\mathbf{L}(\mathbf{w})=0.5 \mathbf{w}^{\mathbf{H}} \mathbf{R}_{\mathbf{x}} \mathbf{w}+\beta\left[1-\mathbf{w}^{\mathbf{H}} \mathbf{a}_{\mathbf{u}}\right]
$$

where $\beta$ is Lagrange multiplier.

$\mathbf{L}(\mathbf{w})$ is a quadratic function of $\boldsymbol{w}$, so the optimum $\boldsymbol{w}$ can be calculated from the equation $\nabla_{w} \mathbf{L}(\mathbf{w})=\mathbf{R}_{\mathbf{x}} \mathbf{w}-\mathbf{a}_{\mathbf{u}} \beta=0$. We have

$$
\mathbf{w}_{\text {opt }}=\mathbf{R}_{\mathbf{x}}^{-1} \mathbf{a}_{\mathbf{u}} \beta
$$

The weight vector must satisfy the constraint equation (6). According to equation (8) and equation (6), we have $\beta=1 /\left(\mathbf{a}_{\mathbf{u}}^{\mathbf{H}} \mathbf{R}_{\mathbf{x}}^{-\mathbf{1}} \mathbf{a}_{\mathbf{u}}\right)$ and

$$
\mathbf{w}_{\text {opt }}=\mathbf{R}_{\mathbf{x}}^{-\mathbf{1}} \mathbf{a}_{\mathbf{u}} /\left(\mathbf{a}_{\mathbf{u}}^{\mathbf{H}} \mathbf{R}_{\mathbf{x}}^{-\mathbf{1}} \mathbf{a}_{\mathbf{u}}\right)
$$

C. Adaptive nulling with main and side lobe constraint

Usually we still want to control the side lobe, and then equation (8) should be modified. Suppose that the weight vector without side lobe constraint is $\mathbf{w}_{\mathbf{0}}$, and the noise covariance matrix is $\mathbf{R}_{n}$, then we can define a new row vector as follow.

$$
\mathbf{R}_{\mathbf{n}} \mathbf{w}_{\mathbf{0}}=\mathbf{e}\left(\mathbf{e}^{\mathbf{H}} \mathbf{R}_{\mathbf{n}}^{-1} \mathbf{e}\right)^{-1}
$$

Based on this equation, we have

$$
\mathbf{e}=\mathbf{w}_{\mathbf{0}}\left(\mathbf{w}_{\mathbf{0}}^{\mathbf{H}} \mathbf{w}_{\mathbf{0}}\right)^{-1}
$$

Comparing equation (10) with (9), we know that the main lobe constraint is not equation (6) any more. The modified main lobe constraint is

$$
\mathbf{w}^{\mathbf{H}} \mathbf{e}=1
$$

And then, we can obtain the optimum transmitting weight vector, which is shown as follow.

$$
\mathbf{w}_{\text {opt }}=\mathbf{R}_{\mathbf{x}}^{-\mathbf{1}} \mathbf{e}\left(\mathbf{e}^{\mathbf{H}} \mathbf{R}_{\mathbf{x}}^{-\mathbf{1}} \mathbf{e}\right)^{-1}
$$

D. Adaptive nulling with main and side lobe constraint and given nulls constraint

We have discussed the method of adaptive nulling in the unknown directions of interferences. However, in the transmitting mode, we not only hope null in the direction of interference, but also hope null in a given direction. So the method of adaptive nulling with main and side lobe constraint and given nulls constraint is discussed as follow.

In this case, when we calculate the optimum weight vector, we should consider not only the main and side lobe constraint, but also the given null constraint. The given null constraint is expressed as

$$
\mathbf{w}^{\mathbf{H}} \mathbf{a}_{g, i}=0, i=1, \cdots, N_{g}
$$

where $\mathbf{a}_{g, i}$ is the directions of given nulls, $N_{g}$ is the number of given nulls.
Now we calculate the optimum weight vector $\mathbf{w}$ with main and side lobe constraint and given nulls constraint, which satisfies that the power output reaches minimum value.

$$
\text { Let } \mathbf{C}=\left[\mathbf{q} a_{g, 1} \cdots a_{g, N_{g}}\right], \quad \mathbf{b}=[10 \cdots 0], \quad \text { here } \mathbf{C} \text { is a }
$$
matrix with dimension of $L \times\left(N_{g}+1\right)$, and $\mathbf{b}$ is a vector with dimension of $1 \times\left(N_{g}+1\right)$. Then the main and side lobe constraint and given null constraint can be rewritten as

$$
\mathbf{w}^{\mathbf{H}} \mathbf{C}=\mathbf{b}
$$

Obviously, there is $N_{p} \leq L-1$. Now we use the Lagrange multiplier approach to solve the this problem. The Lagrange function is defined as follow.

$$
\begin{gathered}
\mathbf{L}(\mathbf{w})=\mathbf{0 . 5} \mathbf{w}^{\mathbf{H}} \mathbf{R}_{\mathbf{x}} \mathbf{w}+\boldsymbol{\beta}\left[\mathbf{b}-\mathbf{w}^{\mathbf{H}} \mathbf{C}\right] \\
\text { From } \nabla_{w} \mathbf{L}(\mathbf{w})=\mathbf{R}_{\mathbf{x}} \mathbf{w}-\mathbf{C} \boldsymbol{\beta}=0 \text {, we have } \\
\mathbf{w}_{\text {opt }}=\mathbf{R}_{\mathbf{x}}^{-\mathbf{1}} \mathbf{C} \boldsymbol{\beta}
\end{gathered}
$$

The optimum $\mathrm{w}$ must satisfy the constraint equation (14). When let (17) in (14), we have $\boldsymbol{\beta}=\left(\mathbf{C}^{\mathbf{H}} \mathbf{R}_{\mathbf{x}}^{-\mathbf{1}} \mathbf{C}\right)^{-\mathbf{1}} \mathbf{b}^{\mathbf{H}}$. And replace $\boldsymbol{\beta}$ in (17), we have

$$
\mathbf{w}_{\text {opt }}=\mathbf{R}_{\mathbf{x}}^{-1} \mathbf{C}\left(\mathbf{C}^{\mathbf{H}} \mathbf{R}_{\mathbf{x}}^{-1} \mathbf{C}\right)^{-1} \mathbf{b}^{\mathbf{H}}
$$

The optimum weight vectors with different constraints are shown in equations (9), (13) and (18) respectively. However, $\mathbf{R}_{\mathbf{x}}$ (or $\mathbf{R}_{\mathbf{I}}$ ) is usually unknown or hard to get. Here we use the sample matrix inversion algorithm to calculate the optimum weight vector. In this algorithm, $\mathbf{R}_{\mathbf{x}}$ is estimated as follow.

$$
\hat{\mathbf{R}}_{X}(N)=\frac{1}{N} \sum_{i=1}^{N} \mathbf{x}(i) \mathbf{x}^{\mathbf{H}}(i)
$$

where $\hat{\mathbf{R}}_{x}(N)$ is the estimation of $\mathbf{R}_{\mathbf{x}}$ of the $N$ th sample. And $\mathbf{x}(i)$ is the array signal of the $i$ th sample.

\section{COMPUTER SIMULATIONS}

Suppose the transmitting Uniform Linear Array has 15 elements, the space between is a half of wave-length, the signal noise ratio (SNR) is $20 \mathrm{~dB}$, sample times are 512 .

(1) Adaptive nulling with main lobe constraint only. The direction of the transmitting main lobe is $0^{\circ}$ on request. Suppose there is only one interference, which direction is $20^{\circ}$. The pattern of adaptive nulling is shown as solid line in Fig.1 (a), the null depth is $-68.7 \mathrm{~dB}$. The dotted line represents the original pattern without nulling. Suppose there are two interferences, which directions are $20^{\circ}$ and $28^{\circ}$. The pattern of adaptive nulling is shown in Fig.1 (b), null depths are -54.8 and $-62.2 \mathrm{~dB}$ respectively.

(2) Adaptive nulling with main and side lobe constraints. The direction of the transmitting main lobe is $0^{\circ}$, and the side lobe is - $30 \mathrm{~dB}$ Dolph-Chebyshev weighted on request. Suppose there is only one interference, which direction is $20^{\circ}$. The pattern of adaptive nulling is shown as solid line in 
Fig.2 (a), the null depth is $-73.3 \mathrm{~dB}$. The dotted line represents the original pattern of $-30 \mathrm{~dB}$ Dolph-Chebyshev weighted without nulling. Suppose there are two interferences, which directions are $20^{\circ}$ and $31^{\circ}$. The pattern of adaptive nulling is shown in Fig.2 (b), the null depths are $-71.2 \mathrm{~dB}$ and $-67.9 \mathrm{~dB}$ respectively.

(3) Adaptive nulling with main and side lobe constraints and given null constraint. The direction of the transmitting main lobe is $0^{\circ}$, and the side lobe is $-30 \mathrm{~dB}$ Dolph-Chebyshev weighted, and there is a given null in the direction of $45^{\circ}$ on request. Suppose there is only one interference, which direction is $20^{\circ}$. The pattern of adaptive nulling is shown as solid line in Fig. 3 (a), the adaptive null depth is $-63.1 \mathrm{~dB}$ and the given null depth is lower than -80dB. Suppose there are two interferences, which directions are $20^{\circ}$ and $31^{\circ}$. The pattern of adaptive nulling is shown in Fig.3 (b), the adaptive null depths are -71.2dB and $-67.9 \mathrm{~dB}$ respectively and the given null depth is lower than $-80 \mathrm{~dB}$.

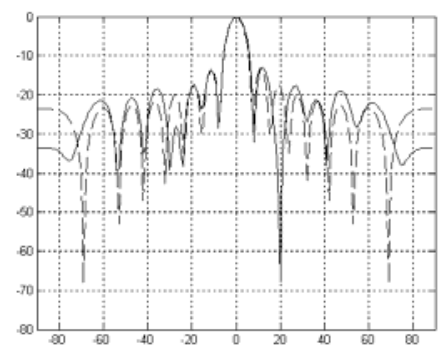

Fig.1(a) Nulling with main lobe constraint (1 interference)

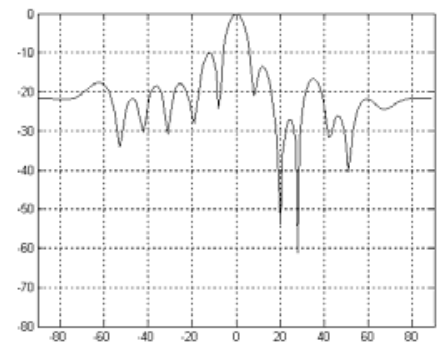

Fig.1(b): Nulling with main lobe constraint (2 interference)

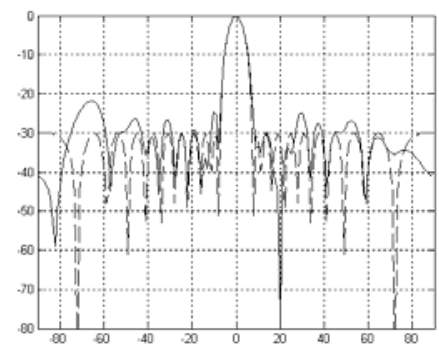

Fig.2(a): Nulling with main and side lobe constraints (1 interference)

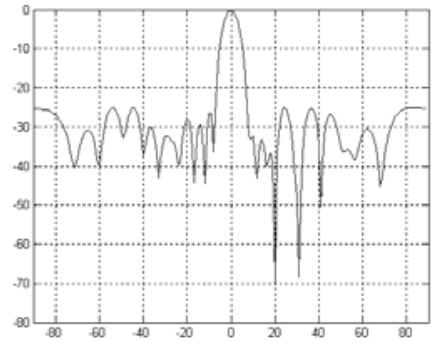

Fig.2(b): Nulling with main and side lobe constraints (2 interference)

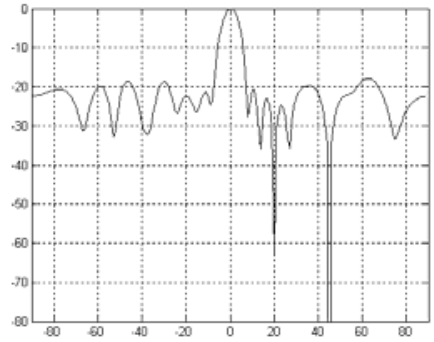

Fig.3(a): Nulling with main and side lobe constraints and given null constraint (1 interference)

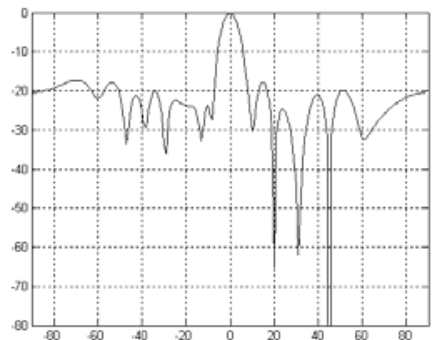

Fig.3(b): Nulling with main and side lobe constraints and given null constraint (2 interference)

\section{CONCLUSIONS}

When we apply DBF to radar and radar EW system, adaptive nulling is very important, which can raise the anti-reconnaissance ability, promote the anti-ARM ability, and avoid the interference of the system. Adaptive nulling methods with multiple constraints for transmitting DBF of ULA are discussed in this paper. The computer simulations show that the proposed methods are practicable and effective.

\section{REFERENCES}

[1] Garrod A. Digital modules for phased array radar[J]. IEEE International Radar Conference, Alexandria. 1995:726-731

[2] S.T. Smith, Optimum phase-only adaptive nulling[J], IEEE Trans. On Signal Processing, 1999, 47(7):1835 1843

[3] H. Steyskal, Digital beamforming at ROME laboratory[J], Microwave Journal, 1996(2):100 126

[4] L. Horowitz, Controlling adaptive antenna arrays with the sample matrix inversion algorithm[J]. IEEE Trans. On AES, 1979.11,15:840 848.

[5] D. J. Rabodeau, P. Parker, Wideband adaptive beamforming to control time sidelobe and null depth, Proc. SPIE,Vol.5077,2003 